\title{
Regulation of Both Cell State and Gene Expression with Nutrient Gradient as Conserved Mechanism Differentiating the Cell Fates for Embryonic Gastrulation: A Theory
}

\author{
Zi-Jian Cai \\ No. 129, Building 6, Room 404, North Dongwu Road, Suzhou, China \\ Email: hrsh8@126.com \\ Received 14 January 2016; accepted 29 January 2016; published 3 February 2016 \\ Copyright (C) 2016 by author and OALib. \\ This work is licensed under the Creative Commons Attribution International License (CC BY). \\ http://creativecommons.org/licenses/by/4.0/ \\ (c) (i) Open Access
}

\begin{abstract}
In this article, it is newly suggested the regulation from nutrient gradient on both cell metabolic state and expressional gene classes as the conserved mechanism differentiating the cell fates for formation of the three germ layers of gastrula in animals. As a new theory, it is supported by the totipotency and pluripotency of early embryonic cells and cultured stem cells to be regulated by nutrients, as well as by the universal formation of nutrient gradient during gastrulation in various animals. Besides, it is also supported by the subsequent phenotypic fates of three germ layers of gastrula, with the endoderm manifesting expression of gene classes in nutritious condition and giving rise to the epithelium of digestive and respiratory system; the ectoderm manifesting expression of gene classes with nutrient dependence and environmental effect, and giving rise to the nervous and epithelial tissues; the mesoderm lying between them and giving rise to the muscle and adipose. Likewise, it is directly supported by the different nutrient effects onto such trilaminar differentiation in various cultured stem cells. Finally, it is in further supported by the evolutionary effects of nutrients in preserving the trilaminar fates in gastrula versus relative permitting the variations in anteroposterior determination. Conclusively, it is identified the nutrient gradient as an important mechanism to partially differentiate the cell fates for embryonic gastrulation.
\end{abstract}

\section{Keywords}

Gastrula, Nutrient, Stem Cell Culture, Gene Expression, Trilaminar Germ Layers, Anteroposterior Determination

Subject Areas: Developmental Biology, Evolutionary Studies, Genetics

How to cite this paper: Cai, Z.-J. (2016) Regulation of Both Cell State and Gene Expression with Nutrient Gradient as Conserved Mechanism Differentiating the Cell Fates for Embryonic Gastrulation: A Theory. Open Access Library Journal, 3: e2348. http://dx.doi.org/10.4236/oalib.1102348 


\section{Introduction}

Embryonic development is a complex and programmed process in animals. How the large quantity of genes are regulated and expressed to program the embryonic development in order has been a difficult topic yet to be solved fully, and attracted the attention of many biologists.

Embryonic zygote forms from the fertilization of an oocyte with a sperm. The zygote divides to form the morula. The cells in further divide and form the blastula with a cavity filled with liquid. Since then, the cells of blastula undergo complex movement and reorganization in various manners varying from species to species, such as invagination, involution, ingression, delamination and epiboly, and form a conserved trilaminar structure called as the gastrula. The conserved three germ layers of gastrula in further undergo organogenesis to generate various organs and complete embryonic development [1].

Zygotes are totipotent and able to divide and produce cells of all differentiated types in animals, while most cells in early embryonic stages are pluripotent. To understand how programmed gene expressions accomplish the complex embryonic development, it is an important step to figure out how genes in the totipotent zygote are regulated in program to form the conserved three pluripotent germ layers of gastrula. In this article, the author integratively analyzes the early embryonic stages in animals, attempting to identify an existent factor directing the gene expressions and differentiating the cell fates for embryonic gastrulation.

\section{Nutrient Regulation of Both Cell State and Gene Expression in Early Embryonic Cells}

The gastrula is universally present in almost all animals except the very primitive coelenterates which are diploblastica. Almost all animals, including platyhelminthes, annelids, arthropods, vertebrates and so on, share the common embryonic stage as gastrula of three layers. Organogenesis begins from the gastrula to generate the various organs in animals [1].

The embryonic stages earlier than gastrula also vary greatly in evolution. For instance, the oocyte in mammals is small in size and develops within the maternal uterus, while the oocyte of Xenopus is big in size and develops independently in water. For another example, the direct-developing sea urchin without larval stage is different from indirect-developing sea urchins with larval stage via extensive remodeling in early stage in localization of maternal determinants in oocyte and dissociation of cell cleavage from axis formation [2]. As the oocytes and mature animals may sometimes adapt independently to different environments in evolution, such as in birds and mammals, the evolutionary changes in oocytes may dissociate from that in their mature animals. This is the feature drawn from the comparative embryology at present.

Even though adaptive and various, the eggs of almost all animals are large in size and heavy in deposition of nutrients, except for mammals which are viviparous. Even in mammals, the embryos in early stages instead absorb nutrients from the maternal blood supply to the uterus, and are certainly also rich in nutrients. Rich in nutrients is the common character of all eggs and zygotes in almost all animals.

All kinds of living cells can detect the concentration of nutrients and respond in their own ways, as easily demonstrated by in vitro culture of cells [3], including pluripotent stem cells [4]. Cells in the early embryonic stages likewise detect and respond to the concentration of nutrients [4]. Difference in concentration of nutrients makes the cells respond in difference and adjust expression of gene classes in difference. In this regard, the nutrients in the early embryonic stages would become the important factors to partially differentiate the cell fates for formation of the three conserved germ layers of gastrula via regulating both cell metabolic state and expressional gene classes in totipotent and pluripotent embryonic cells. This deduction is new and provable, so that becomes a new theory.

The totipotency of zygotes and pluripotency of embryonic cells in early embryonic stages supports this theory. Due to the totipotency and pluripotency, the zygotes and early embryonic cells would certainly respond to the concentration of nutrients, partially differentiating their cell fates via regulating both their cell metabolic state and expressional gene classes. It has been shown that in vitro culture of pluripotent stem cells indeed detected and responded to nutrients [4]. For comparison, it was also reported that the maternal corticosterone was transferred into avian yolk and able to alter offspring growth and adult phenotype [5]. Whereas, it was unclear whether the effect resulted from the modulation in early embryonic stages, or later after the necessary expression of receptors of corticosterone. However, with nutrients present in almost all animal eggs or early embryonic stages, it is certain that, in contrary to corticosterone, the universally present nutrients in zygotes are the natural 
important factors to partially differentiate the cell fates via regulating both cell metabolic state and expressional gene classes during the early embryonic stages for formation of the gastrula.

\section{Nutrient Gradient as Generally Formed to Partially Differentiate the Cell Fates for Embryonic Gastrulation}

It is necessary to point out that, as mentioned above, even though all eggs or zygotes of animals are supplied rich in nutrients, they vary greatly in forms of morphology, patterns of division and processes of invagination in various species in evolution. Whereas, the three germ layers of gastrula are conserved in evolution. For the various forms of zygotes to partially differentiate into the pluripotent cells in the conserved three layers in gastrula, it is necessary to differentiate the concentration of nutrients in a conserved way during gastrulation. Nutrient gradient is exactly the simple conserved form to differentiate the concentration of nutrients for gastrulation. In the followings, it is comprehensively inspected the various forms of eggs or zygotes, while it is adopted the emerged universal nutrient gradient to in further support the proposed theory for directing the partial differentiation during gastrulation.

In some zygotes, such as in birds, the distribution of nutrients is not symmetrical in the early embryos, so that the early embryogenesis makes use of the existent gradient of nutrients to direct the cell fates for gastrulation [6]. In mammals, even though the zygotes are mostly homogeneous, the embryos absorb the nutrients as gradient from the maternal blood supply, so that also adopt the gradient of nutrients to direct the cell fates for formation of the three layers of gastrula in accordance.

Nonetheless, the eggs of amphibian Xenopus laevis are relatively homogeneous and only partially affected by gravity. It was reported that the eggs of Xenopus laevis fertilized on the orbiting Space Shuttle were able to produce nearly normal larvae, even though manifesting some abnormalities in early embryogenesis [7]. Whereas, it has also been reported that the localized Hermes regulated the cleavage of specific vegetally derived cell lineages in Xenopus laevis [8]. Obviously, differentiation in rate of cell proliferation generated the difference in content of nutrients versus the size of nucleus in various Xenopus embryonic cells [8] [9]. In this way, the relatively homogeneous zygotes of Xenopus laevis also realize the differentiation in concentration of nutrients as gradient to direct the embryonic cell fates during the formation of three conserved layers of gastrula.

Asymmetrical cleavage gives rise to two daughter cells of different sizes, and certainly generates difference in the ratio of nutrient contents versus nuclear sizes in daughter cells. Asymmetrical cleavage occurs in many spiral holoblastic organisms, such as in the early embryo of leech Helobdella robusta [10]. In accordance, besides differentiation in rate of cell proliferation, asymmetry in division of cells also helps differentiate the concentration of nutrients into gradient during early embryogenesis, and directs the partial differentiation in gastrulation.

Centrolecithal eggs are special in that they place the yolk in the centre of their cytoplasm. Many arthropod eggs are centrolecithal. Centrolecithal zygotes undergo meroblastic cleavage, where the cleavage plane extends only to the accumulated yolk and is superficial [11]. Direction of cell fates with nutrient gradient can only occur later after asymmetrical allocation of the generated cell nuclei, after which there leads to partial differentiation in gastrulation.

Some transcriptional factors present in albumin, yolk and blastoderm in zygotes have also been reported to be able to regulate gene expressions [12]. However, nutrients are densely deposited or sufficiently supplied to the early embryonic cells, so that are concentrated as signals and factors for regulation of metabolic state and gene classes of cells. Some other embryonic regulatory factors in early embryonic stages, such as the transcriptional factors, would only cooperate with rather than counteract against the dominant signaling roles of concentrated nutrients universally present as gradient. For example, as mentioned above, it has been reported that the localized Hermes functioned to regulate the cleavage of specific vegetally derived cell lineages in Xenopus laevis [8], which certainly needed to cooperate with the concentrated nutrients there in vegetal half.

It is necessary to point out that, after gastrulation, organogenesis starts [1] and the embryo begins to form its own circulatory system. Glucose gradually becomes the main form of nutrient for embryonic cells. While at the stages earlier than gastrula, without the embryonic blood vessels, it is the proteins, lipids and polysaccharides that are the main forms of nutrients for embryos. Mammals are exceptional as viviparous, so that glucose is the direct maternal nutrient that is supplied to the embryo at the earliest stage.

It is noted here that recent achievements of molecular neutral evolution have expanded from molecular level to higher interactive levels. It has been demonstrated that, besides synonymous substitutions as neutral drift, weak nonsynonymous substitutions were interactive at higher levels and also occurred frequently as near-neutral 
in evolution [13]. With the nutrient gradient as the key differentiating factor in regulating the pluripotent cell states and directing the cell fates for gastrulation, there would permit many nonsynonymous near-neutral substitutions in the early embryonic stages, manifesting near-neutral in microevolution and natural selection in macroevolution.

In all, nutrient gradient is suggested as the important factor or mechanism to direct the partial differentiation of cell fates for formation of the conserved three layers of gastrula via regulating both cell metabolic state and expressional gene classes. It is supported by the totipotency of zygotes and pluripotency of early embryonic cells, as well as the response of cultured stem cells to nutrients. Nutrients form gradient in concentration during gastrulation via asymmetrical distribution of nutrients or blood flow of supply in some animals. Besides, nutrients also form gradient during gastrulation via differentiation in rate of cell proliferation or asymmetry in division of cells in other animals. In these ways, the nutrients universally differentiate in concentration into gradient to partially differentiate the cell fates for formation of the conserved three germ layers of gastrula.

\section{Phenotypic Fates of Three Germ Layers in Gastrula}

During early embryonic development, zygote cleavage generates the morula and then blastula. Embryonic cells of blastula undergo complex movement and invagination, and generate the gastrula of conserved trilaminar structure. The three germ layers of gastrula are the endoderm, ectoderm and mesoderm. At this stage, intercellular inductive signals other than nutrients begin to affect [1]. The gastrula starts organogenesis to generate various organs [1].

Generated through several early stages from zygote to morula and blastula, the cells in gastrula have acquired partial differentiation, becoming pluripotent from totipotent. As the partial differentiation of cells in the conserved three germ layers of gastrula is the consequence of the postulated mechanism for direction of cell fates with nutrient gradient, its outcomes can also be adopted to prove the correctness of the new theory.

The endodermal cells in gastrula lie innermost in embryo, and most adjacent to the nutrients in gradient. To adapt to the nutritious condition, the pluripotent cells must express genes to digest the nutrients, and synthesize glucose, amino acids and so on to supply to other embryonic cells. In this regard, the metabolic state and gene expression in these cells directed or regulated by nutrients are coordinated toward this fate for cells, which imprints onto the subsequent organogenesis. During organogenesis, the endodermal cells in gastrula indeed differentiate and give rise to the epithelial lining of the digestive tract, associated glands and respiratory system in vertebrates [14], as well as in Drosophila [15]. In this respect, the regulation of both cell state and gene classes in endoderm with proposed nutrient gradient during gastrulation fits well with the phenotypic outcome of development, supporting the rationality of the theory.

The ectodermal cells in gastrula lie outmost in embryo, and most distant to nutrients in gradient. To adapt to the relatively outmost environment, the pluripotent cells must rely more on other cells for nutrient supply, and express genes for sensation of and protection against the relatively environmental effect, such as more keratins, primitive neuronal characters and so on. The metabolic state and gene expression in these cells directed or regulated by the nutrient dependence and environmental effect are coordinated toward this fate for cells, and imprint onto the subsequent organogenesis. During organogenesis, the cells of embryonic ectoderm give rise to neural progenitors in the dorsal side and epidermal progenitors in the ventral side in vertebrates [16], while also give rise to neural cells [17] and epidermal cuticle in Drosophila [18]. In this regard, the regulation of both cell state and gene classes in ectoderm with proposed nutrient gradient during gastrulation fits with the developmental outcome of phenotype, also supporting the rationality of the theory.

The mesodermal cells in gastrula lie between the endoderm and ectoderm. To adapt to the moderate condition, the pluripotent cells must express genes to absorb nutrients and store energy, and so on. The metabolic state and gene expression in these cells directed or regulated by moderate nutrients and conditions are coordinated toward this fate for cells, and imprint onto the subsequent organogenesis. During organogenesis, the cells of embryonic mesoderm give rise to bones, muscles [19], and adipose tissues [20] in vertebrates, and to muscles in Drosophila [21]. Therefore, the regulation of both cell state and gene classes in mesoderm with proposed nutrient gradient during gastrulation fits with the generated phenotypes, in further supporting the rationality of the theory.

It is necessary to point out that, in addition to the partial trilaminar differentiation of embryonic cells in response to nutrient gradient in gastrula, the mutual interaction of cells or gastrula induction also begins to effect [1]. However, due to the totipotency and pluripotency of early embyronic cells, with the nutrients as concentrated regulatory signals, these mutual inductions are overcome or suppressed from counteracting against the 
concentrated nutrients in effects. Besides, the mutual inductions need partial trilaminar differentiation as prerequisite which is ultimately caused by the gradient of nutrients. In this regard, mutual inductions are not in contradiction with the identification of nutrient gradient herein as an existent and sometime even an ultimate mechanism to partially differentiate the cell fates for the three germ layers of gastrula.

Moreover, cultures of stem cells even directly manifest the trilaminar differentiation of embryonic cells in response to nutrient gradient. It was reported that activation of amino acid response through limitation of them in culture favored the primitive endodermal, visceral endodermal, and endodermal lineages, whereas suppressed the mesodermal and certain ectodermal lineages [22], demonstrating the endodermal tendency to transform and supply nutrients. In contrary, cultured Xenopus presumptive ectoderm was made successful in long term in a nutrient-supplemented culture medium [23], while cultured neural progenitor cells were usually promoted in proliferation in vitro with hypoxia [24] [25], demonstrating the ectodermal direction to use extrinsic nutrients and respond to environmental stress. Besides, mesoderm-specific transcript was shown to be associated with fat mass expansion in response to positive energy balance [26], suggesting the mesodermal direction to use nutrients and store energy. In these respects, stem cell cultures strongly supported the gradient of nutrients as a mechanism to regulate the metabolic state of embryonic cells, directing the endodermal cells to transform and supply nutrients, the mesodermal cells to use nutrients and store energy, as well as the ectodermal cells to rely on nutrient supply and respond to environmental stress.

It is noted that some growth factors have been shown to be necessary for the morphogenesis of mesoderm in early gastrula [27] [28]. As these factors are present in the early embryonic liquid and can reach all three layers of gastrula on the same relative position in anteroposterior gradient, it is the differentiation of cells in nutritious gradient and their mutual inductions that differentiate the cellular response of the three layers of gastrula, with only the middle layer transformed into the mesoderm. The roles of these growth factors can only cooperate with rather than counteract against that of the universally present nutrient gradient.

\section{Nutrient Preserving the Trilaminar Fates in Gastrula versus Permitting the Variations in Anteroposterior Determination}

One of the important achievements in molecular development is the identification of many genes and molecules necessary for the generation of anteroposterior pattern in various animals. The contrast between conservation in trilaminar fates in gastrula and relative variation in determination of anteroposterior pattern supplies additional support to the theory that the nutrient gradient is an important factor to direct the cell fates for the three germ layers of gastrula.

Drosophila is well studied for the molecular mechanisms related to the generation of anteroposterior polarity. Early, it was found that the segmented pattern of Drosophila embryo was organized by two activities localized at the anterior and posterior egg poles, with both activities inducing and polarizing the pattern [29]. Recently, it has been identified that these activities in eggs were maternal genes encoding for proteins getting translated upon fertilization to establish concentration gradients spanning the egg. Bicoid and Hunchback are the maternal genes that are important for patterning of anterior parts of Drosophila embryo [30]-[32], while Nanos and Caudal are maternal effect genes that are important for the formation of more posterior abdominal segments of Drosophila [32].

Vertebrates are different from Drosophila in mechanisms to determine the anteroposterior polarity. Rather than maternal deposition of anteroposterior polarity in eggs in Drosophila, the anteroposterior ectodermal patterning in Xenopus occurs by the onset of gastrulation [33] [34], while the perpendicular gradients of Wnt and BMP determine the anteroposterior and dorsoventral body axes [35].

It is common knowledge that Drosophila and other arthropods are protostome animals, while Xenopus and other vertebrates are deuterostome. Protostome and deuterostome animals are reverse in anteroposterior polarity. It is an ancient and drastic divergence in evolution to separate these two basic forms of animal patterns. Hence, it is not surprising that there are differences in mechanisms in the present protostome and deuterostome animals for determination of their anteroposterior polarity, such as in Drosophila and Xenopus illustrated here.

The variations in mechanisms to determine the anteroposterior polarity are more frequent in occurrence within vertebrate embryos than the simple divergence of protostome and deuterostome animals. Analyses with amphibian embryos demonstrated that the induction and anteroposterior patterning of the central nervous system was initiated by signals produced by the organizer and organizer-derived axial mesoderm [33] [34]. Whereas, it 
was nonetheless shown that the initial anteroposterior pattern of the central nervous system in zebrafish depended on the differential competence of the epiblast and was not imposed by organizer-derived signals [36]. Obviously, the mechanisms for determination of anteroposterior polarity varied more frequently in vertebrates in evolution.

In contrast, the developmental fates of the three germ layers of gastrula are quite conserved in evolution. The endoderm is destined to differentiate into the epithelial lining of digestive tract, associated glands and respiratory system in both vertebrates [14] and Drosophila [15]. The ectoderm is destined to differentiate into the neural progenitors and epidermal progenitors in vertebrates [16], and to neural cells [17] and epidermal cuticle in Drosophila [18]. The mesoderm is destined to differentiate into the bones, muscles [19], and adipose tissues [20] in vertebrates, and to muscles in Drosophila [21].

The evolutionary preservation in trilaminar fates in gastrula versus variation in anteroposterior determination suggests there must be present a conservative factor or mechanism during embryonic gastrulation to partially differentiate the cell fates into the three germ layers in gastrula. Nutrient gradient is the suitable factor or mechanism universally present during embryonic gastrulation which fits for this purpose. This is an additional support to the theory that regulation with nutrient gradient on cell state and gene expression is the mechanism to partially differentiate the cell fates for formation of three conserved germ layers of gastrula.

\section{Conclusions}

In this article, it is proposed a new theory that regulation from nutrient gradient on both cell state and gene expression is the evolutionarily conserved mechanism to partially differentiate the cell fates for formation of the three germ layers of gastrula.

The theory is supported by the totipotency and pluripotency of early embryonic cells to be regulated by nutrients as concentrated signals during the early embryonic stages, as well as supported by the universal formation of nutrient gradient during gastrulation in various animals. It is additionally supported by the phenotypic fates of three germ layers of gastrula, with the endodermal cells responding to nutritious condition and giving rise to the epithelium of digestive tract, associated glands and respiratory system in both vertebrates and Drosophila; the ectodermal cells relying on extrinsic nutrients and responding to environmental effects, and giving rise to the neural and epidermal cells in vertebrates and Drosophila; the mesoderm lying between the endoderm and ectoderm, and giving rise to the muscle and adipose tissues in vertebrates, and muscles in Drosophila. Nutrient and oxygen manipulations in various stem cell cultures are also consistent with the trilaminar differentiation in cell fates, and even directly support the nutrients as key regulatory factors in embryonic gastrulation.

The variations in mechanisms in determination of anteroposterior polarity occurred more frequently in vertebrates than the divergence of protostome and deuterostome animals in evolution. The preservation in trilaminar fates in gastrula versus the relative variation in anteroposterior determination also supports the theory that the nutrient gradient is likely the natural conservative factor or mechanism to partially differentiate the cell fates during embryonic gastrulation while relatively permit other evolutionary variations.

In all, with this theory supported with many comparative embryologic studies and stem cell cultures, it is herein identified the nutrient gradient as the key and universal factor or mechanism to partially differentiate the cell fates for formation of the three germ layers of gastrula.

\section{Perspectives}

Since the nutrient gradient is identified as the conserved mechanism to differentiate the cell fates for formation of the three germ layers of gastrula in animals, it is herein revealed a simple and universal mechanism in the early developmental stage of animals, and unified the animal developmental mechanisms at early stages with evolution.

In future, more investigations would be needed to reveal the detailed genetic regulation by the universally present nutrient gradient during gastrulation. While, in vitro experiments with various stem cells on this issue would also be beneficial to biomedical research of tissue regeneration.

\section{Disclosures}

The author declares that there is no other financial support, nor conflict of interest for this paper. 


\section{References}

[1] Cai, Z.-J. (2015) Termination of Organogenesis as Intrinsic Constraint on Animal Development and Evolution: A Theory. Open Access Library Journal, 2, e1646. http://dx.doi.org/10.4236/oalib.1101646

[2] Raff, R.A. (1992) Direct-Developing Sea Urchins and the Evolutionary Reorganization of Early Development. Bioessays, 14, 211-218. http://dx.doi.org/10.1002/bies.950140403

[3] Eagle, H. (1955) Nutrition Needs of Mammalian Cells in Tissue Culture. Science, 122, 501-514. http://dx.doi.org/10.1126/science.122.3168.501

[4] Baker, M. (2011) Stem Cells in Culture: Defining the Substrate. Nature Methods, 8, 293-297. http://dx.doi.org/10.1038/nmeth0411-293

[5] Hayward, L.S. and Wingfield, J.C. (2004) Maternal Corticosterone Is Transferred to Avian Yolk and May Alter Offspring Growth and Adult Phenotype. General and Comparative Endocrinology, 135, 365-371. http://dx.doi.org/10.1016/j.ygcen.2003.11.002

[6] Callebaut, M., Harrisson, F. and Bortier, H. (2001) Effect of Gravity on the Interaction between the Avian Germ and Neighbouring Ooplasm in Inverted Egg Yolk Balls. European Journal of Morphology, 39, 27-38. http://dx.doi.org/10.1076/ejom.39.1.0027

[7] Black, S., Larkin, K., Jacqmotte, N., Wassersug, R., Pronych, S. and Souza, K. (1996) Regulative Development of Xenopus laevis in Microgravity. Advances in Space Research, 17, 209-217. http://dx.doi.org/10.1016/0273-1177(95)00637-T

[8] Zearfoss, N.R., Chan, A.P., Wu, C.F., Kloc, M. and Etkin, L.D. (2004) Hermes Is a Localized Factor Regulating Cleavage of Vegetal Blastomeres in Xenopus laevis. Developmental Biology, 267, 60-71. http://dx.doi.org/10.1016/j.ydbio.2003.10.032

[9] Chan, A.P. and Etkin, L.D. (2001) Patterning and Lineage Specification in the Amphibian Embryo. Current Topics in Developmental Biology, 51, 1-67. http://dx.doi.org/10.1016/S0070-2153(01)51001-7

[10] Weisblat, D.A. (2007) Asymmetric Cell Divisions in the Early Embryo of the Leech Helobdella robusta. Progress in Molecular and Subcellular Biology, 45, 79-95. http://dx.doi.org/10.1007/978-3-540-69161-7 4

[11] Zissler, D. (1992) From Egg to Pole Cells: Ultrastructural Aspects of Early Cleavage and Germ Cell Determination in Insects. Microscopy Research and Technique, 22, 49-74. http://dx.doi.org/10.1002/jemt.1070220106

[12] Knepper, P.A., Mayanil, C.S., Hayes, E., Goossens, W., Byrne, R.W. and McLone, D.G. (1999) The Presence of Transcription Factors in Chicken Albumin, Yolk and Blastoderm. In Vitro Cellular \& Developmental Biology-Animal, 35, 357-363. http://dx.doi.org/10.1007/s11626-999-0087-4

[13] Ohta, T. (2002) Near-Neutrality in Evolution of Genes and Gene Regulation. Proceedings of the National Academy of Sciences of the United States of America, 99, 16134-16137. http://dx.doi.org/10.1073/pnas.252626899

[14] Fukuda, K. and Kikuchi, Y. (2005) Endoderm Development in Vertebrates: Fate Mapping, Induction and Regional Specification. Development, Growth \& Differentiation, 47, 343-355. http://dx.doi.org/10.1111/j.1440-169X.2005.00815.x

[15] Nakagoshi, H. (2005) Functional Specification in the Drosophila Endoderm. Development, Growth \& Differentiation, 47, 383-392. http://dx.doi.org/10.1111/j.1440-169X.2005.00811.x

[16] Chang, C. and Hemmati-Brivanlou, A. (1998) Cell Fate Determination in Embryonic Ectoderm. Journal of Neurobiology, 36, 128-151. http://dx.doi.org/10.1002/(SICI)1097-4695(199808)36:2<128::AID-NEU3>3.0.CO;2-3

[17] Stüttem, I. and Campos-Ortega, J.A. (1991) Cell Commitment and Cell Interactions in the Ectoderm of Drosophila melanogaster. Development, Suppl. 2, 39-46.

[18] Payre, F. (2004) Genetic Control of Epidermis Differentiation in Drosophila. The International Journal of Developmental Biology, 48, 207-215. http://dx.doi.org/10.1387/ijdb.15272387

[19] Pourquié, O. (2001) Vertebrate Somitogenesis. Annual Review of Cell and Developmental Biology, 17, 311-350. http://dx.doi.org/10.1146/annurev.cellbio.17.1.311

[20] Han, J., Lee, J.E., Jin, J., Lim, J.S., Oh, N., Kim, K., Chang, S.I., Shibuya, M., Kim, H. and Koh, G.Y. (2011) The Spatiotemporal Development of Adipose Tissue. Development, 138, 5027-5037. http://dx.doi.org/10.1242/dev.067686

[21] Nguyen, H.T. and Frasch, M. (2006) MicroRNAs in Muscle Differentiation: Lessons from Drosophila and Beyond. Current Opinion in Genetics \& Development, 16, 533-539. http://dx.doi.org/10.1016/j.gde.2006.08.010

[22] Shan, J., Hamazaki, T., Tang, T.A., Terada, N. and Kilberg, M.S. (2013) Activation of the Amino Acid Response Modulates Lineage Specification during Differentiation of Murine Embryonic Stem Cells. American Journal of Physiology_Endocrinology and Metabolism, 305, E325-E335. http://dx.doi.org/10.1152/ajpendo.00136.2013

[23] Fukui, Y., Furue, M., Myoishi, Y., Sato, J.D., Okamoto, T. and Asashima, M. (2003) Long-Term Culture of Xenopus 
Presumptive Ectoderm in a Nutrient-Supplemented Culture Medium. Development, Growth \& Differentiation, 45, 499506. http://dx.doi.org/10.1111/j.1440-169X.2003.00717.x

[24] Hegewald, C., Alt, R., Hetz, S., Cross, M., Acikgoez, A., Till, H., Metzger, R. and Metzger, M. (2011) Reduced Oxygen Stress Promotes Propagation of Murine Postnatal Enteric Neural Progenitors in Vitro. Neurogastroenterology and Motility, 23, e412-e424. http://dx.doi.org/10.1111/j.1365-2982.2011.01761.X

[25] Zhu, L.L., Zhao, T., Huang, X., Liu, Z.H., Wu, L.Y., Wu, K.W. and Fan, M. (2011) Gene Expression Profiles and Metabolic Changes in Embryonic Neural Progenitor Cells under Low Oxygen. Cellular Reprogramming, 13, 113-120. http://dx.doi.org/10.1089/cell.2010.0043

[26] Nikonova, L., Koza, R.A., Mendoza, T., Chao, P.M., Curley, J.P. and Kozak, L.P. (2008) Mesoderm-Specific Transcript Is Associated with Fat Mass Expansion in Response to a Positive Energy Balance. The FASEB Journal, 22, 3925-3937. http://dx.doi.org/10.1096/fj.08-108266

[27] Adomako-Ankomah, A. and Ettensohn, C.A. (2014) Growth Factors and Early Mesoderm Morphogenesis: Insights from the Sea Urchin Embryo. Genesis, 52, 158-172. http://dx.doi.org/10.1002/dvg.22746

[28] Ciruna, B. and Rossant, J. (2001) FGF Signaling Regulates Mesoderm Cell Fate Specification and Morphogenetic Movement at the Primitive Streak. Developmental Cell, 1, 37-49. http://dx.doi.org/10.1016/S1534-5807(01)00017-X

[29] Nüsslein-Volhard, C., Frohnhöfer, H.G. and Lehmann, R. (1987) Determination of Anteroposterior Polarity in Drosophila. Science, 238, 1675-1681. http://dx.doi.org/10.1126/science.3686007

[30] Chang, C.W., Nashchekin, D., Wheatley, L., Irion, U., Dahlgaard, K., Montague, T.G., Hall, J. and St Johnston, D. (2011) Anterior-Posterior Axis Specification in Drosophila Oocytes: Identification of Novel Bicoid and Oskar mRNA Localization Factors. Genetics, 188, 883-896. http://dx.doi.org/10.1534/genetics.111.129312

[31] Driever, W. and Nüsslein-Volhard, C. (1989) The Bicoid Protein Is a Positive Regulator of Hunchback Transcription in the Early Drosophila Embryo. Nature, 337, 138-143. http://dx.doi.org/10.1038/337138a0

[32] Lasko, P. (2011) Posttranscriptional Regulation in Drosophila oocytes and Early Embryos. Wiley Interdisciplinary Reviews: RNA, 2, 408-416. http://dx.doi.org/10.1002/wrna.70

[33] Gamse, J. and Sive, H. (2000) Vertebrate Anteroposterior Patterning: The Xenopus neurectoderm as a Paradigm. Bioessays, 22, 976-986. http://dx.doi.org/10.1002/1521-1878(200011)22:11<976::AID-BIES4>3.0.CO;2-C

[34] Slack, J.M. and Tannahill, D. (1992) Mechanism of Anteroposterior Axis Specification in Vertebrates. Lessons from the Amphibians. Development, 114, 285-302.

[35] Pera, E.M., Acosta, H., Gouignard, N., Climent, M. and Arregi, I. (2014) Active Signals, Gradient Formation and Regional Specificity in Neural Induction. Experimental Cell Research, 321, 25-31. http://dx.doi.org/10.1016/j.yexcr.2013.11.018

[36] Koshida, S., Shinya, M., Mizuno, T., Kuroiwa, A. and Takeda, H. (1998) Initial Anteroposterior Pattern of the Zebrafish Central Nervous System Is Determined by Differential Competence of the Epiblast. Development, 125, 1957-1966. 